\title{
Constant Refractive Index Multi-Core Fiber Laser
}

R.J. Beach, M.D. Feit, L.D. Brasure, S.A. Payne, R.W. Mead, J.S. Hayden, D. Krashevich, S.A. Alunni

This article was submitted to Optical Society of America Advanced Solid State Lasers Seventeenth Topical Meeting and Tabletop Exhibit, Quebec City, Canada, February 3-6, 2002

\section{March 18, 2002}

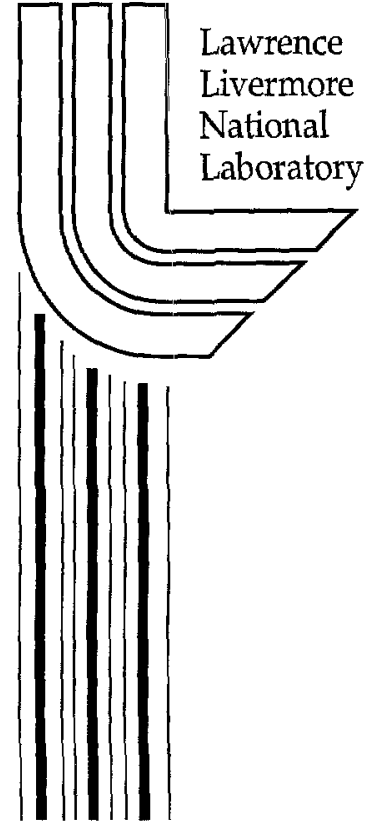




\section{DISCLAIMER}

This document was prepared as an account of work sponsored by an agency of the United States Government. Neither the United States Government nor the University of California nor any of their employees, makes any warranty, express or implied, or assumes any legal liability or responsibility for the accuracy, completeness, or usefulness of any information, apparatus, product, or process disclosed, or represents that its use would not infringe privately owned rights. Reference herein to any specific commercial product, process, or service by trade name, trademark, manufacturer, or otherwise, does not necessarily constitute or imply its endorsement, recommendation, or favoring by the United States Government or the University of California. The views and opinions of authors expressed herein do not necessarily state or reflect those of the United States Government or the University of California, and shall not be used for advertising or product endorsement purposes.

This is a preprint of a paper intended for publication in a journal or proceedings. Since changes may be made before publication, this preprint is made available with the understanding that it will not be cited or reproduced without the permission of the author.

This report has been reproduced directly from the best available copy.

Available electronically at http://www.doe.gov/bridge

Available for a processing fee to U.S. Department of Energy and its contractors in paper from

U.S. Department of Energy

Office of Scientific and Technical Information

P.O. Box 62

Oak Ridge, TN 37831-0062

Telephone: (865) 576-8401

Facsimile: (865) 576-5728

E-mail: reports@adonis.osti.gov

Available for the sale to the public from

U.S. Department of Commerce

National Technical Information Service

5285 Port Royal Road

Springfield, VA 22161

Telephone: (800) 553-6847

Facsimile: (703) 605-6900

E-mail: orders@ntis.fedworld.gov

Online ordering: http://www.ntis.gov/ordering.htm

OR

Lawrence Livermore National Laboratory Technical Information Department's Digital Library

http://www.llnl.gov/tid/Library.html 


\title{
Constant Refractive Index Multi-Core Fiber Laser
}

\author{
Raymond J. Beach, Michael D. Feit, LeAnn D. Brasure*, and Stephen A. Payne \\ Lawrence Livermore National Laboratory, PO Box 808, Livermore, CA 94551 \\ Phone: (925)423-8986, FAX: (925)423-6195, email: beach2@llnLgov
}

\author{
Richard W. Mead \\ Collimated Holes, Inc. \\ 460 Division Street \\ Campbell, CA 95008
}

\author{
Joseph S. Hayden, David Krashkevich, and David A. Alunni \\ Schott Glass Technologies, Inc. \\ 400 York Avenue, Duryea, PA 18642
}

\begin{abstract}
A scalable fiber laser approach is described based on phase-locking multiple gain cores in an antiguided structure. The waveguide is comprised of periodic sequences of gain-and no-gainloaded segments having uniform index, within the cladding region. Initial experimental results are presented.
\end{abstract}

(C) 2002 Optical Society of America

OCIS codes: (230.7370) Waveguides; (060.2320) Fiber optics amplifiers and oscillators

\section{Introduction}

We have defined a new power-scalable concept for fiber lasers known as the Ribbon Laser. The strategy for scaling is to include numerous cores in a single fiber, each able to deliver a certain amount of power (eventually about $100-200 \mathrm{~W}$ ). The key, of course, is to maintain a phase-locked wave front across these numerous core regions.

A simplified drawing of our Ribbon Laser, having only one transverse dimension, appears in Fig. 1 , where a transverse profile is shown. In this example, the refractive index is plotted, and it is seen to vary periodically. The two main approaches for achieving phase-locking are based on: evanescent and radiative coupling. If the effective index of the propagating mode is intermediate between the low and high indices, then the coupling is evanescent (i.e. the "tails" of the neighboring fields in each of the cores overlap). If the effective index is lower, then the field is not confined and migrates freely throughout the entire Ribbon structure.
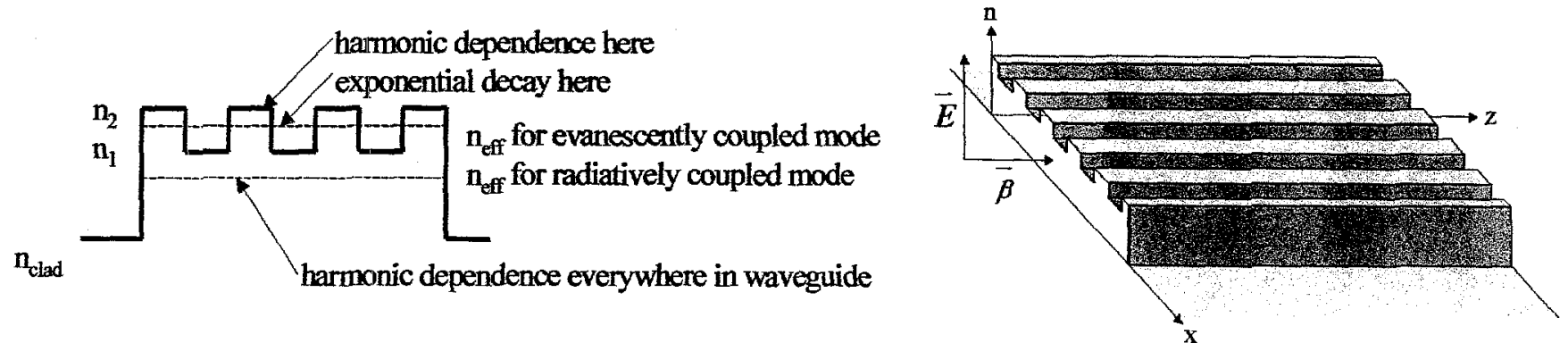

Fig. 1 LHS: Simplified drawing of the transverse Ribbon Laser structure, showing the modulated refractive index, and the $n_{\text {eff }}$ values that give rise to evanescent and radiatively coupled modes. $R H S$ : Three-dimensional drowing of E-field, propagation direction $(+z)$, and refractive index.

We have found that a "photonic bandgap" emerges in this structure, depending on the sense of the refractive index variation. ${ }^{1}$ In Fig. 2 below, we see that the gain regions are the same in the upper and lower plots, although the modulation of the index has an opposite sign. On the LHS, the overlap for each of the modes is plotted against the effective index, while the fields characterizing the two modes on each

\footnotetext{
'Presently at Air Force Research Laboratory, Albuquerque, NM
} 
side of the bandgap are expressly shown on the RHS. It is readily apparent that the bandgap is analogous to the valence and conduction bands of a crystal.
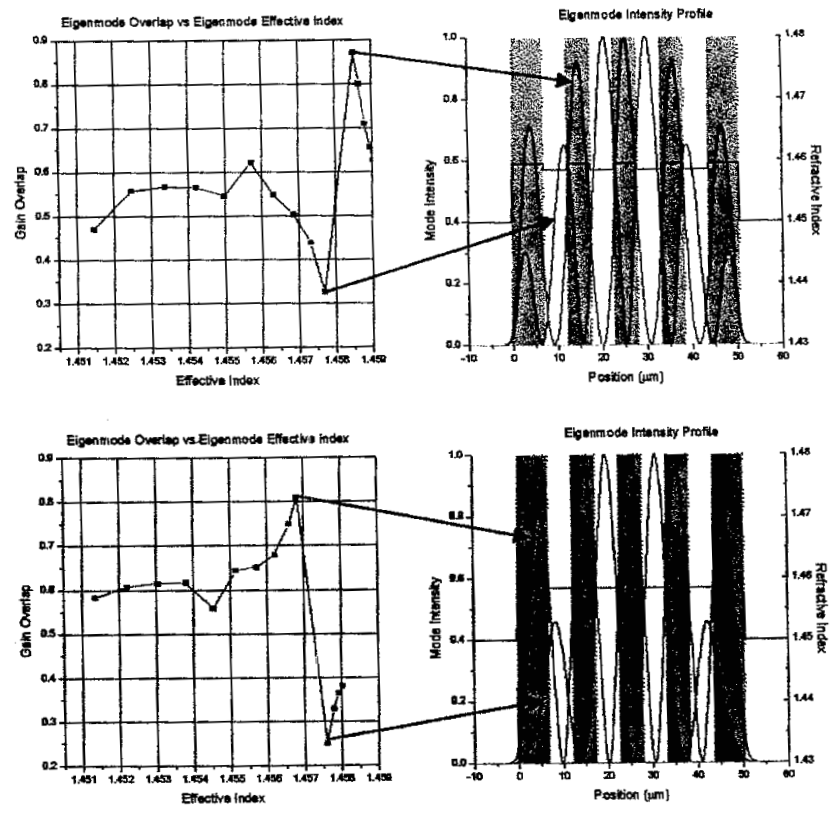

Fig. 2 Plots of the overlap of the field with the gain regions, against the effective index of the modes. The field intensities for the two modes at the "photonic bandgap" are plotted on the right.

The most interesting result that we obtained is pictured below in Fig. 3. Here we utilize a constant index across the Ribbon Laser, and observe that the mode structure leads to the dominance of a single mode that has considerably more overlap than all the other modes. Of course the greater overlap of this mode leads to much higher gain, such that it becomes the preferred operating mode of the laser. The preferred mode exhibits five intensity peaks, one within each of the gain regions. All of the other modes have more or less than five intensity lobes, so they have less overlap with the gain regions. This type of structure, based on having a uniform index across the ribbon structure, is analogous to a "photonic metal," since the uniform refractive index is like the constant potential seen by electrons in a metal.

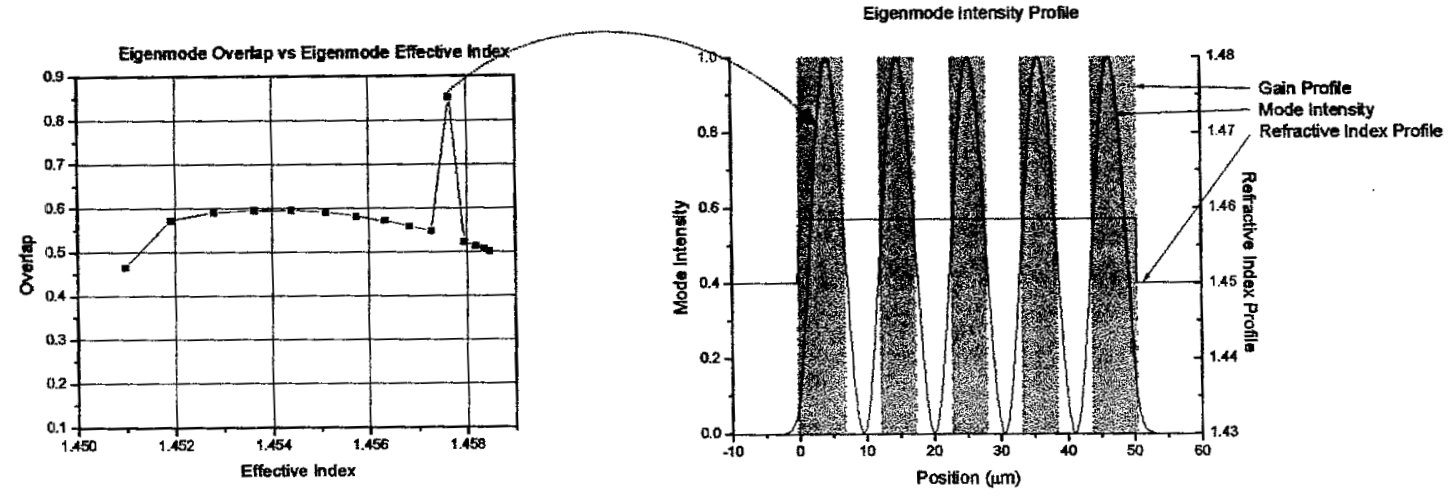

Fig. 3 Predicted modal structure for 5-core Ribbon Laser, based on a uniform transverse refractive index profile. A single preferred mode emerges that has significantly more overlap (and gain) than the other modes.

The one-dimensional modeling outlined above gives insight into the occurrence of desired anti-guided leaky modes in structures of the type considered here. In order to understand the impact of two transverse dimensions on the performance of realistic structures, we have considered a number of designs with either rectangular or circular cores that either touch the boundary or are immersed in the inner cladding region. For this analysis, we have employed a full scalar-wave propagation method to simulate device 
performance. As an example, a cross section of a five-gain core ribbon laser structure is shown in Fig. 4 along with its preferred mode near field intensity.

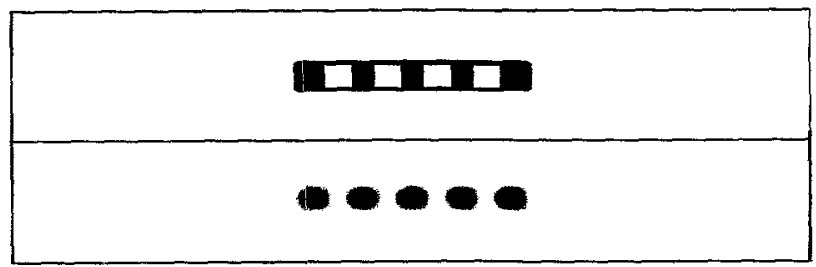

Fig. 4 Cross sectional view of two-dimensional ribbon structure. The dark regions in the upper picture indicate the gain-loaded portions of the waveguide. The refractive index is constant throughout the waveguide region. The near field output intensity of the highest gain mode is shown in the lower panel.

For constant index waveguide regions the leaky modes have a pure sinusoidal character. This implies the far field will principally consist of two lines with an angular spread of $\pm \lambda / \mathrm{p}$ in the horizontal direction. Here $\lambda$ is the wavelength in vacuum and $p$ is the transverse period. Similarly, the angular spread in the vertical direction is determined by the structure height. Our numerical simulations confirm these expectations. Figure 5a shows the simulated far field pattern for the ribbon structure of Fig. 4. Because the modal field is coherent, collimation can be achieved by use of a phase plate. Additionally, since the modal structure is so simple, the most convenient approach is to adjust the phases of periods of the structure by either $\mathbf{0}$ or $\pi$, successively, to effectively yield the absolute value of the modal field. This elimination of zero crossings greatly improves the far field pattern as shown in Fig. $5 \mathrm{~b}$ where the simulation reveals that $2 / 3$ of the modal power has been concentrated in a central peak. The horizontal width of this peak depends on the number of cells in the structure and will improve as $1 / \mathrm{N}$ for the ideal structure.

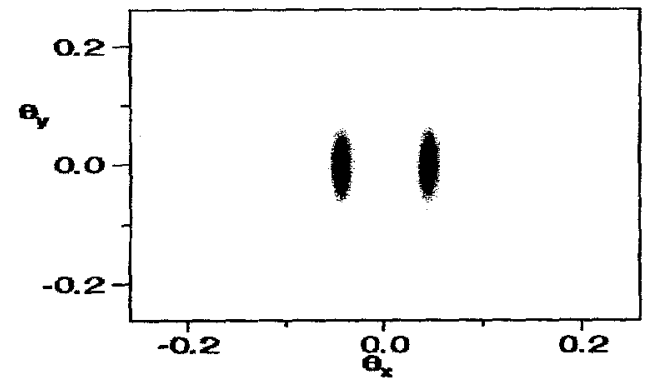

(a)

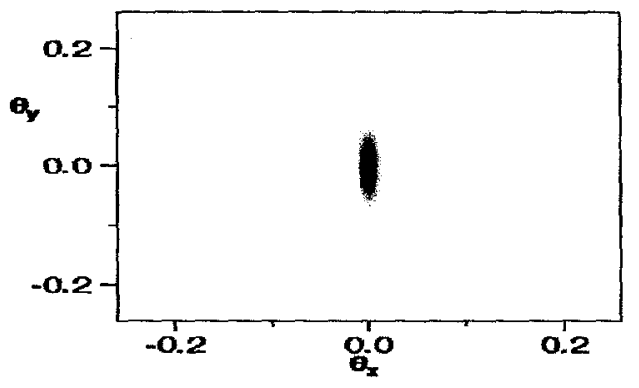

(b)

Fig. 5 Far field of the ribbon structure shown in Fig. 4 (a) before and (b) after correction with simple phase plates. Approximately $2 / 3$ of the modal power in (b) is concentrated in the central peak.

The ultimate goal of this modeling is to develop an understanding of robust fiber ribbon designs that will guarantee single transverse mode operation in the presence of strong gain saturation. As such, it is useful to determine the tolerances on cell refractive indices for the various cells that comprise a given structure. To answer this question we investigate systematic errors in cell refractive indices that represent aspects of the ribbon structure fabrication that are beyond the limit of control and which could be an issue with the technology we will use to fabricate prototype structures. The results of this study, which are plotted in Fig. 6 , show the overlap of the largest and second largest modes for structures with varying numbers of gain cores. These data were generated using our one-transverse-dimension model. It is evident in Fig. 6 that as the number of cells increases, the requirements on the systematic variation of the refractive index become more stringent. For the 5-core case, the index control must be within $0.001-0.002$, a value that is achievable with soft glasses. 


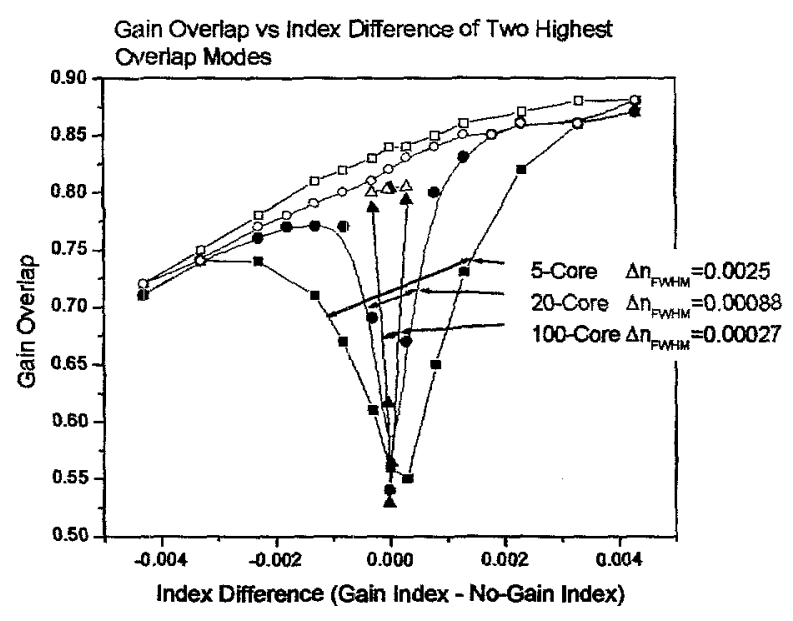

Fig. 6 Overlap vs. index between the gain and no-gain regions. Three different structures are investigated here consisting of 5,20 , and 100 cores, respectively. The gain overlap of the highest overlap and next highest overlap mode are plotted against the index variation.

\section{Prototype Ribbon Fiber Structures}

Our proposed approach offers a pathway for scaling fiber lasers to the high average power regime based on phase locking multiple gain cores in a fiber structure using anti-guiding or radiative coupling. The initial focus is on a ribbon-like geometry surrounded by a circular pump cladding as shown in Fig. 7, in which the waveguide region contains 5 gain cores alternating with non-gain regions in a periodic array. An outer, lower index cladding surrounds the entire ribbon structure. The distinguishing feature of the Ribbon Laser design is a constant refractive index profile across the waveguide region as opposed to alternating higher and lower index regions. As indicated above, our modeling predicts that the constant index design will provide a mode spectrum that meets two critical design requirements: (1) strongly favored oscillation in a single transverse mode, and (2) good intensity uniformity across the waveguide structure. We require single mode operation so that a static phase corrector placed in the near field of the ribbon laser's output can optimize the phase across the aperture to achieve a high Strehl output beam. The second requirement is necessary to ensure that the gain saturates in a uniform manner across the structure so as not to increase the propensity of the device to operate in multiple transverse modes. Conceptually, the device can be scaled to higher powers simply by increasing the number of anti-guided cores within the ribbon. Modeling predicts this design will scale to 100 embedded gain cores with reasonable tolerances requirements if fused silica is used as the base material. The strong phase locking inherent in this approach should allow all the gain cores in the ribbon to communicate with each other and therefore scale to higher powers with higher phase fidelity across the aperture than is possible with similar evanescently coupled structures. This approach, in which the gain elements are radiatively coupled in a "leaky" waveguide array, is analogous to the most successful scheme for phasing laser diode elements. ${ }^{2}$

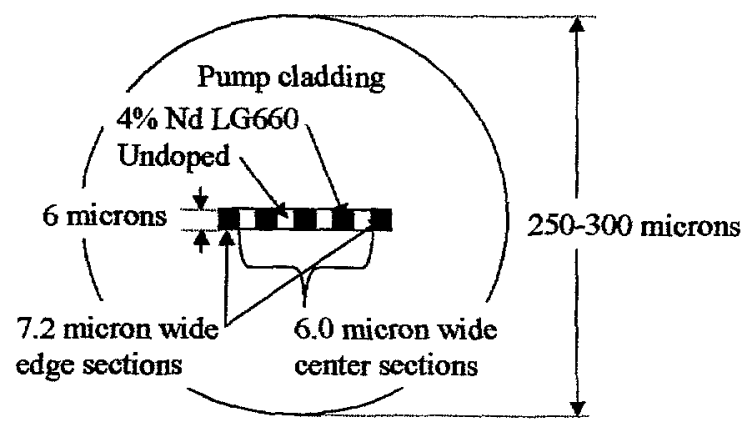

Fig. 7 Cross-sectional view of 5-core ribbon fiber being fabricated with Schott glasses. The central rectangular region is the waveguide region and the larger circular region is the pump cladding. 
The structure shown in Fig. 7 uses 5 gain cores and a waveguide region that is $\sim 6 \mu \mathrm{m}$ high by $\sim 60 \mu \mathrm{m}$ wide. The pump radiation is confined to the larger $\sim 300 \mu \mathrm{m}$ diameter circular outer clad. As a material for this prototype device we have selected LG-660, an alkali-zinc-silicate laser glass, as the base glass. LG-660 offers viscosity characteristics compatible with redraw technology without risk of devitrification. A production melt of LG- 660 doped with $4 w t \% \mathrm{Nd}_{2} \mathrm{O}_{3}$ serves as the active core glass. Passive lanthanum containing variants of LG-660 were prepared to serve as undoped core and cladding glasses. The undoped core glass matched the active glass in refractive index and thermal expansion to $<0.001$ and $<1 \times 10^{-7} / \mathrm{K}$ respectively. On the other hand, the composition of the undoped cladding glass was adjusted to provide an index $0.006-0.007$ less than the core glasses, and with a thermal expansion roughly $2 \times 10^{-7} / \mathrm{K}$ lower than the core glasses, insuring that during fabrication of redrawn ribbon the cladding glass would be placed under compression.

\section{Experimental Results}

In collaboration with Schott Glass Technologies and Collimated Holes, we have produced a first prototype of the Ribbon Laser structure (shown below in Fig. 8). While the index control is barely acceptable at this juncture (the doped regions in the waveguide have an index value that is larger than the undoped regions by .0012), the device has exhibited a reasonable level of coherence to suggest that our theory of the Ribbon Laser is viable.

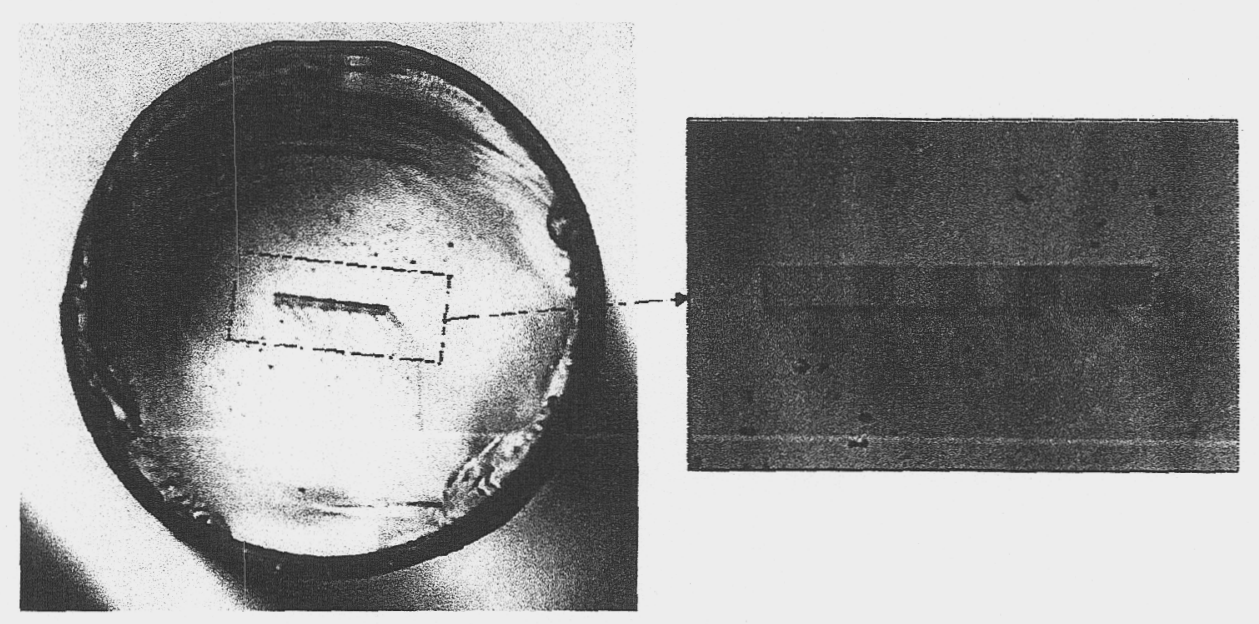

Fig. 8 Photograph of the first prototype Ribbon Laser, showing the outer pump clad, and the alternating $N d$-doped and undoped regions.

The prototype Ribbon Laser exhibited optical to optical efficiencies of up to $20 \%$, as noted in Fig. 9. The laser operated near $1.05 \mu \mathrm{m}$ as expected, and was pumped near $0.808 \mu \mathrm{m}$. Also featured in Fig. 9 (RHS) are measurements of the coherence exhibited by the device. In these experiments, two of the various cores (numbered 1 through 5) are arranged to interfere - the depth of the modulation or the visibility of the fringes is seen to vary from $39 \%$ to $13 \%$. With improved index control, we expect to attain the full level of modulation indicating a single phase coherent mode is lasing across the entire aperture. 

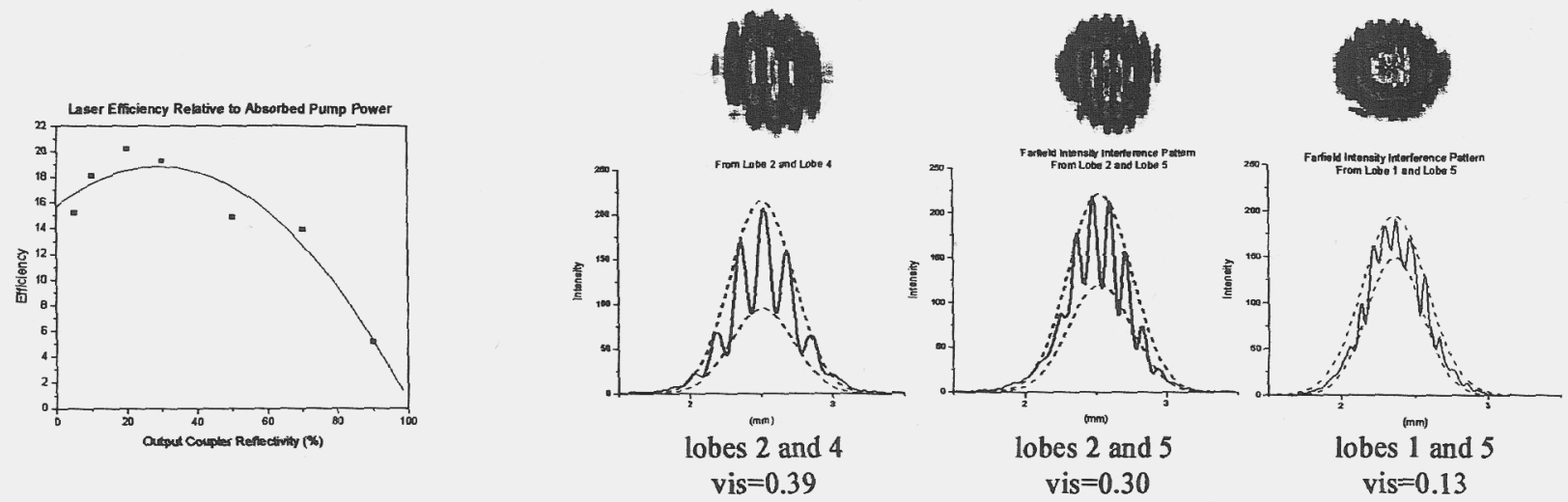

Fig. 9 Experimental data obtained from the first prototype Ribbon Laser, including slope efficiency data and measurements of the fringe visibility (coherence) between pair of cores.

At this juncture, we have completed our theory of the Ribbon Laser and have demonstrated that the first working prototype has properties consistent with predictions. With these initial results we are encouraged that the Ribbon Laser concept is scalable, and could one day reach power levels consistent with laser weapons. An improved prototype device is currently in testing.

\section{Summary}

In summary, we have developed and are in the process of demonstrating a new, robustly scalable technique for phase locking multiple gain cores in a fiber structure based on anti-guiding or radiative coupling. We have selected and extensively modeled a ribbon-like geometry in which the waveguide region contains multiple gain cores alternating with non-gain regions imbedded in a constant index profile, yielding good mode selectivity and intensity uniformity across the waveguide structure. Initial experimental demonstrations and characterizations are focused on a five-gain core ribbon structure. This work was performed under the auspices of the U.S. Department of Energy by the University of California Lawrence Livermore National Laboratory under contract No. W-7405-Eng-48, and by the Air Force Research Laboratory.

\section{References}

${ }^{1}$ R.J. Beach, M.D. Feit, R.H. Page, L.D. Brasure, R. Wilcox, and S.A. Payne, "Scalable Antiguided Ribbon Laser Concept", JOSA B accepted for publication.

${ }^{2}$ D. Botez and D. R. Scifres, Diode Laser Arrays, Chapter 1, Cambridge Univ. Press 1994. 